\section{José María}

Arguedas y

la Revista

Pumaccabua:

creación literaria

e investigación

etnográfica

Nécker Salazar Mejía
Recebido em: 28 de outubro de 2019

Aceito em: 18 de novembro de 2019
Investigador y docente universitario, es doctor en literatura peruana $y$ latinoamericana por la Universidad Nacional Mayor de San Marcos. Editó el libro Churata desde el Sur. Actúa como profesor de la Facultad de Humanidades de la Universidad Nacional Federico Villarreal, Perú, y de la Unidad de Posgrado de la Facultad de Letras y Ciencias Humanas de la Universidad Nacional Mayor de San Marcos, Perú. Contato:nsalazar@unfv.edu.pe Peru 
PALABRAS CLAVE: José María Arguedas; revista Pumaccahua; educación indísena; literatura tradicional; cultura andina

KEYWORDS: José María Arguedas; Pumaccahua masazine; Andean education; traditional literature; Andean culture
Resumen: El artículo estudia la experiencia educativa desarrollada por José María Arguedas cuando se desempeñaba como profesor en el Colegio Nacional Mateo Pumaccahua, de Sicuani, en el Cusco, entre los años de 1939 y 1941 . El trabajo analiza la concepción pedagósica que puso en práctica, que se distinguió por el impulso de la creación literaria y la investigación etnográfica. El escritor despertó en los alumnos el susto literario, la escritura creativa, la lectura crítica y el interés por las manifestaciones de la cultura andina. Producto de esta experiencia fue la publicación de la revista Pumaccahua, que reúne poesías, comentarios de textos, breves ensayos y descripciones de tradiciones y costumbres locales realizados por los estudiantes. El trabajo de Arguedas en Sicuani demuestra que el protagonismo de los alumnos es el núcleo fundamental de la educación y que la escuela puede ser un lugar propicio para la creación, la reflexión y la investigación.

Abstract: This paper studies the pedasogical experience developed by José María Arguedas when he worked as a teacher at the Mateo Pumaccahua National School, in Sicuani, in Cusco, between 1939 and 1941. The work analyzes the pedasogical conception that Arguedas implemented, which was distinguished by the impulse of literary creation and ethnographic research. In this way, he involved the students into the literary taste, creative writing, critical reading, as well as their interest in the manifestations of the Andean culture. Product of his experience was the publication of Pumaccahua magazine, which brings an insight into poetry, text comments, short essays and exhibitions of local traditions and customs created by the students themselves. The work of Arguedas in Sicuani demonstrates that the student's role is the fundamental core of education and that the school can be propitious place for creation, reflection and research. 


\section{INTRODUCCIÓN}

José María Arguedas (1911-1969), narrador, poeta, antropólogo y traductor, es uno de los mayores representantes del indigenismo en el Perú. Nacido en Andahuaylas, fue hablante monolingüe del quechua y aprendió el castellano en su niñez. En Lima, logró forjarse una sólida formación en la Universidad Nacional Mayor de San Marcos, trabó amistad con poetas, artistas e intelectuales, y desarrolló una intensa actividad promocionando la música, el folclor y las manifestaciones culturales de los pueblos indios del Perú. En su producción literaria, conformada, entre otras obras, por Agua, Yawar fiesta, Los ríos profundos y Todas las sangres, confluyen dos culturas en una relación de permanente confrontación. A su importante obra de creación se suman sus investigaciones antropológicas, centradas en el estudio del arte musical, la danza, el cuento oral, las festividades locales y las costumbres y tradiciones de las comunidades indias y mestizas, que contribuyen a un mayor conocimiento de la cultura y del mundo andino.

Arguedas fue también docente de educación básica en varios colegios secundarios: en el Colegio Nacional Mateo Pumaccahua, de Sicuani, en el Cusco, entre los años de 1939 y 1941; en el Colegio Nacional Alfonso Ugarte, de Lima, entre 1942 y 1944, y en el Colegio Nacional Nuestra Señora de Guadalupe, de Lima, entre 1944 y 1948. Por otro lado, invitado por Emilio Barrantes, el escritor trabajó en el Ministerio de Educación en 1941 y conformó la Comisión de Reforma de los Planes de Estudio; en dicho ministerio, fue designado Conservador general de Folklore y Jefe de la Sección de Folklore, Bellas Artes y Despacho, en 1947 y 1952, respectivamente. 
Arguedas tuvo una especial vocación por la educación, se interesó por innovar los programas curriculares y por mejorar la calidad de la enseñanza y de los textos escolares. Es muy reconocida su importante labor en la difusión y promoción de las manifestaciones folclóricas y del arte tradicional.

En el Colegio Mateo Pumaccahua, la concepción pedagógica de Arguedas se distinguió por fomentar la lectura, estimular la creación literaria, impulsar la investigación etnográfica y despertar el interés por la recopilación de las tradiciones locales de los pueblos de la sierra del Perú. Dicha experiencia pedagógica se plasma en la revista Pumaccahua, publicada en 1940, que se halla conformada por los trabajos de los propios alumnos'. La publicación demuestra que es posible articular los objetivos de la educación de acuerdo con los intereses de los estudiantes y con los principios de la escuela activa, que promueve un aprendizaje real y significativo.

\section{La práctica educativa de Arguedas y su Relación con Experiencias Pedagógicas del SIGLO XX}

La importancia de la práctica educativa de Arguedas ${ }^{2}$ se puede apreciar mejor en relación con las experiencias y propuestas pedagógicas que se

1 La primera edición de la revista Pumaccahua se publica como un folleto en Sicuani, Cusco, en 1940; la publicación está incluida en Nosotros los maestros (2011), libro editado por Wilfredo Kapsoli que reúne los escritos de Arguedas sobre asuntos pedagógicos. También se encuentra reproducida en el tomo 2 de su Obra antropológica (2012). Para el presente trabajo, las citas de la revista se toman de la segunda edición, que es una edición facsimilar, publicada por la Casa de la Literatura Peruana el año 2018.

2 Un amplio trabajo sobre la trayectoria docente y el pensamiento pedagógico de Arguedas es desarrollado por Wilfredo Kapsoli (2011) en "El maestro José María Arguedas", que es el estudio preliminar del libro Nosotros los maestros. 
desarrollan en las primeras décadas del siglo XX en otras regiones del Perú. Así, en Puno José Antonio Encinas pone en práctica los principios de la "Escuela nueva", a través de los cuales impulsa una educación articulada con la tradición, la historia, la realidad, los valores y las necesidades del indio. El emblemático Centro Escolar $N^{\circ} 881$, donde Encinas fue director, representa una verdadera propuesta de cambio del sistema de enseńanza y de los cimientos de una concepción de la escuela que debía ser superada. De acuerdo con los planteamientos expuestos en Un ensayo de Escuela nueva en el Perú (1932), la educación es parte indesligable de la propiedad de la tierra y tiene un sentido integral; además, debe tener un vínculo con el trabajo, así como una proyección hacia el pueblo.

Otra experiencia importante es desarrollada en Huancayo por Emilio Barrantes, que es muy similar al trabajo que Arguedas lleva a cabo en Sicuani. Tal como lo explica Carmen María Pinilla (2013), Barrantes promueve entre sus alumnos del Colegio Santa Isabel de Huancayo la recopilación de tradiciones populares con el fin de recuperar la "historia viva" y la "riqueza de la propia cultura". Producto de este trabajo es la publicación del folleto Folklore de Huancayo (1940), que es un antecedente de la revista Pumaccahua, cuyo contenido comprende costumbres, canciones, danzas, supersticiones, leyendas, tradiciones y cuentos de la literatura regional. En dicha publicación, Barrantes sostiene que es relevante "la investigación minuciosa de la vida popular en sus manifestaciones características" y que es necesario obtener documentos "que sean trasunto mismo del indio, que copien su vida íntima, la 
tonalidad de sus emociones, el cauce y la dirección de sus sentimientos" (1940, 79-80).

En otro plano, Arguedas cultiva una fructífera amistad con el mexicano Moisés Sáenz, quien conocía nuestra realidad social y cultural, y estaba interesado en la educación peruana, en particular, en la temática indígena. Sáenz invita a Arguedas a participar en el Primer Congreso Indigenista que se desarrolla en Pátzcuaro en 1940, lo que le permitió a nuestro escritor tener un mayor conocimiento de los problemas que planteaban la educación indígena y la escuela rural ${ }^{3}$. Pinilla precisa que fue de mucha importancia "para Arguedas conocer las discusiones que se dieron entonces sobre la alfabetización del adulto y el bilingüismo, las funciones de la escuela rural y las relaciones entre la escuela y la comunidad" $(2013,23)$.

La práctica pedagógica de Arguedas guarda estrecha relación con las concepciones educativas que surgen en el curso del siglo XX. Al respecto, Hugo González (2011) explica que las propuestas del escritor concuerdan con los principios del paidocentrismo y de la escuela activa, con los postulados de la teoría sociocultural basada en el contexto, así como con los planteamientos del aprendizaje significativo. Desde un enfoque más reciente, situándonos

3 Además de Arguedas, también asistieron como invitados por el Perú José Antonio Encinas e Hildebrando Castro Pozo. El congreso, que congregó la participación de representantes de México, Perú, Chile, Bolivia, Ecuador, Guatemala, Colombia, Panamá y El Salvador, alcanzó bastante éxito. Sus acuerdos proponían la reivindicación de los derechos indígenas y la transformación de las condiciones socioeconómicas de las comunidades: "reconocimiento de la propiedad individual y colectiva de la tierra, mantenimiento y uso de las lenguas, educación especial, legislación protectora y respeto de las costumbres (...) aprovechamiento de las instituciones tradicionales, protección y promoción de las artes (...)" (Giraudo, 2011, 41). 
en los postulados de la pedagogía moderna, la experiencia de Arguedas se enmarca dentro de la corriente denominada “investigación-acción”, ya que "parte de situaciones problémicas". González sostiene que, desde esta corriente, se resalta "la figura principal del docente como investigador y no como un transmisor o repetidor de conocimientos, donde solo se ejercita la memoria o el almacenamiento de datos, alejada de la reflexión, meditación, crítica y creatividad" $(2014,174)$.

\section{La experiencia de Arguedas en el Colegio Nacional Mateo Pumaccahua}

El año de 1939, Arguedas es nombrado profesor de Castellano y Geografía en el Colegio Nacional Secundario de Varones Mateo Pumaccahua en Sicuani, Cusco, donde permanece hasta octubre de 1941. Dicha estancia fue tal vez "el momento más feliz de su vida", como lo recuerda el propio escritor. Su experiencia educativa en el Colegio Pumaccahua tiene un especial significado, porque lo convierte en un verdadero promotor de la literatura y del folclor regional. Esta labor es un buen ejemplo de la relación positiva entre el docente y el alumno, así como de las enormes posibilidades de una educación basada en el desarrollo de la capacidad imaginativa de los estudiantes.

Los alumnos del Colegio Pumaccahua eran adolescentes y jóvenes de procedencia india y mestiza, cuya lengua materna era el quechua y cuyas edades oscilaban entre los 14 y 20 ańos. Entre los profesores, se encontraba el Padre Jorge Lira, conocedor de la lengua quechua y recopilador de la literatura 
oral, con quien Arguedas cultivó una gran amistad y compartió la misma inclinación por los estudios de la cultura andina y la literatura quechua ${ }^{4}$.

En los años en que Arguedas desarrolla su actividad docente, Sicuani era una ciudad que se hallaba en proceso de modernización debido al impacto del ferrocarril, el desarrollo de la industria y la actividad comercial. Además, Sicuani se había convertido en el eje de un circuito que vinculaba a las provincias del Cusco, Arequipa y Puno. Juan Alberto Osorio refiere que Sicuani vivía una intensa actividad cultural, teatral y literaria en esos años, que era promovida por poetas, escritores e intelectuales. La lectura de revistas y semanarios extranjeros, los programas de radio y la creación artística distinguían a la ciudad en la primera mitad del siglo XX. De este modo, "[c]uando José María Arguedas llega a Sicuani encuentra una ciudad con una tradición literaria y cultural" (Osorio, 2018, párr.11).

La publicación de la revista Pumaccahua en 1940 testimonia los frutos de la concepción educativa de Arguedas, quien revela su entusiasmo por el trabajo de los alumnos en sus correspondencias con Manuel Moreno Jimeno y Emilio Adolfo Westphalen, en las que les comenta el proceso de gestación de dicha publicación5. La propuesta del autor de Agua enfatiza el

4 El Padre Lira realizó una importante recopilación de un aproximado de sesenta cuentos quechuas en el distrito de Maranganí, ubicado en el Vilcanota. Arguedas ofrece la traducción al castellano de una selección de las narraciones en su libro Canciones y cuentos del pueblo quechua (1949). Entre los textos, destacan conocidos cuentos de la literatura oral andina, como "El torito de la piel brillante", "La amante de la culebra" y "El negociante de harinas".

5 En una carta que data de agosto de 1939, Arguedas le escribe a Moreno Jimeno sobre el trabajo con sus alumnos: "Estoy haciendo trabajar a los muchachos. No te imaginas cuán feliz soy constatando las formidables posibilidades de estos muchachos mestizos. (...) están recolectando 
valor y la importancia de una educación contextualizada que incentive el estudio de la realidad local:

Hacer memorizar a los alumnos las innumerables cuestiones que el plan señala -como hablarles de idiomas romances a los del primer año- tenía su importancia. Pero lo fundamental me parecía, o por lo menos de igual importancia que el estudio de los cursos de año, conseguir que los alumnos empezaran ya a estudiar la región o Provincia donde funcionara el Colegio, y los pueblos de origen de los alumnos (Arguedas, 2018, 1).

En el pensamiento de Arguedas, es indispensable el conocimiento de la comunidad y el vínculo de la escuela con sus tradiciones culturales. Igualmente, es imprescindible ahondar en los valores de los pueblos estudiando sus expresiones vitales y su historia. Para el novelista, la responsabilidad formadora y social de la escuela se asienta en la investigación de las manifestaciones culturales y los saberes de las comunidades, y en su divulgación:

(...) me parecía evidente que los colegios, con una labor así, cumplirían una indispensable función nacional, en un país donde todo, o casi todo, está por investigarse todavía, y donde, por consiguiente, la divulgación de datos sobre la realidad espiritual de los pueblos de las distintas regiones del país y acerca de la riqueza geográfica de estas regiones es indispensable, tanto para los estudiosos, como para todos los alumnos del Perú (Arguedas, 2018, 1).

todo el folklore de la región. (...) Cuando pueda editar mi revista ha de ser algo verdaderamente valioso" (Forgues, 1993, 60). En una carta escrita a Westphalen, fechada el 16 de julio de 1939, Arguedas le comenta la calidad de los escritos de sus alumnos y la diversidad de información reunida por ellos: "Leyendo esos trabajos, hubo ratos en que poco me faltó para llorar de alegría. Son de un valor incalculable, como arte y como documento. Tengo unos 40 para publicarlos. (...). No sólo tengo poemas y otros artículos. Tengo trabajos de los alumnos sobre danzas, costumbres, waynos, leyendas, cuentos; y descripción de pueblos, de ríos, de cerros, lagunas. Tengo trabajos sobre economía, sobre el trigo, el maíz, habas, etc. ...Cuando pueda sacar mi Revista, será algo muy bueno; para los que buscan arte, y para los que quieren datos" (Westphalen Ortiz, 2011, 54-55). 
Las ideas de Arguedas en torno a la educación se adscriben al paradigma de la escuela activa que motiva el aprendizaje creativo, reflexivo y participativo de los estudiantes. El escritor tiene fe en el cambio que se puede fomentar a través de la educación si los docentes asumen con convicción nuevas estrategias y formas de enseñanza; en especial, si impulsan a los alumnos hacia la investigación: “(...) el despertar en los alumnos la inquietud de investigar por cuenta propia, es decir, el despertar en la conciencia del alumno una íntima y profunda necesidad de saber, y un interés exigente de conocer a su país" $(2018,1)^{6}$.

\section{El método de trabajo de Arguedas en Sicuani}

En principio, Arguedas motivaba a los estudiantes y los orientaba sobre el valor de realizar trabajos etnográficos. Al trazar su plan de actividades de campo, el escritor y antropólogo proyectaba el trabajo educativo hacia la comunidad. Cabe precisar que la investigación, el recojo de fuentes y la recopilación de los diferentes testimonios que realizaban los alumnos se

6 En el artículo "El rastro de Arguedas", publicado en el suplemento Variedades de El Peruano, el 23 de mayo de 2011, José Vadillo Villa nos ofrece el testimonio de Guillermo Cáceres Villa, de 85 años entonces, quien fuera estudiante del Colegio Pumaccahua: "Yo soy el único de los alumnos de Arguedas que subsiste en toda la provincia de Canchis". Cáceres Villa, discípulo del novelista entre 1939 y 1940, relata con orgullo que "esa promoción fue una generación importante para Sicuani: uno de ellos llegó a alcalde; otro fue, en su momento, el único abogado de toda Canchis, y hubo poetas, escritores, periodistas, profesores" $(2011,5)$. Vadillo Villa también reproduce entrevistas a profesores y directivos del colegio, quienes valoran el aporte del escritor en el campo de la educación bilingüe e intercultural. 
hacían fuera del aula ${ }^{7}$ El trabajo de campo consistía en la observación y registro de las actividades artísticas y culturales producidas a nivel local y regional; para ello, los alumnos se encargaban de la explicación y la descripción del hecho observado; igualmente, de hacer las transcripciones de los relatos orales. Por indicación del docente, en el desarrollo del trabajo, los estudiantes podían recurrir a diversos sistemas de representación -no solo la escrita- como dibujos y fotografías. De este modo, el alumno se entrenaba en diversas formas de aprendizaje y en el dominio de procedimientos y actividades de carácter práctico.

Luego de haber recogido el material de campo, los alumnos leían sus informes y explicaban el resultado de sus investigaciones; producto de ello, se hacían debates en el aula, lo que complementaba el recojo de las fuentes. Arguedas explica que los intercambios de ideas eran tan intensos que duraban horas, con lo que se propiciaba una importante discusión en torno al propio trabajo realizado por los estudiantes. El aula de clases se convertía entonces en un escenario ideal en el que se desarrollaba en forma horizontal una positiva relación profesor-alumno, en el marco de un fructífero diálogo entre culturas y concepciones del mundo ${ }^{8}$. Sobre este punto, Raúl Jurado

7 En su testimonio, Cáceres Villa recuerda que Arguedas "[n]o era un docente ordinario ni gustaba de encerrarse en el aula”; por el contrario, buscaba un vínculo directo de los estudiantes con el entorno local: "Salvo que una lluvia lo impida, le gustaba caminar por los cerros; nos llevaba a una fiesta, a una feria y nos decía, ‘¿describan!’ Y ahí mismo nos calificaba” (Vadillo Villa, 2011, 6).

8 Cáceres Villa nos ofrece un retrato de Arguedas como docente en Sicuani. "El más destacado profesor que tuvo el Colegio Pumaccahua. Fue un docente fuera de serie, amaba nuestro folklore, se solazaba con nuestro paisaje, peruanista y andino por antonomasia, enseñaba la asignatura de Lenguaje en forma vivencial, no a base de definiciones, ni ejercitando la memoria. El llevaba 
afirma que "[1]a relación de la educación con la etnoliteratura en Arguedas es un signo de rescate permanente del imaginario cultural andino" $(2003,75)$.

Los textos recogidos por los alumnos, conjuntamente con sus creaciones, constituyen el cuerpo de la revista Pumaccahua. Fanni Muñoz afirma que, con dicha publicación, "se refleja el esfuerzo del maestro por estimular la capacidad de investigar la creatividad artística entre sus estudiantes" (2005, 193). Esta promoción de la capacidad expresiva de los alumnos alienta una mayor relación afectiva con el docente, quien ve en el esfuerzo de sus alumnos el objetivo cumplido de una educación orientada a impulsar el talento y el poder de creación de sus propios estudiantes?. La experiencia de Arguedas es una labor relevante que se encuadra "dentro de una perspectiva que concede la máxima importancia a motivar al estudiante y valorar sus producciones intelectuales" (Muñoz, 2005, 193).

La revista Pumaccahua es, igualmente, asumida como un importante material de clases, ya que es empleado por el autor como parte del desarrollo de los cursos que imparte en el colegio. De este modo, el producto de los estudiantes se convierte en un recurso didáctico, pertinente y adecuado, que evidencia su utilidad práctica y su valor académico. Daniel Mathews sostiene que esta preocupación por el propio proceso de aprendizaje de los

a contemplar la naturaleza, para allí tener que inspirarnos un verso o escribir una narración, composición, redacción o descripción y así surgieron extraordinarios vates...” (2012, 60).

9 Tres años después de su experiencia en Sicuani, Arguedas también pone en práctica la misma metodología motivando la creación literaria entre sus alumnos del Colegio Guadalupe de Lima a través de narraciones basadas en la experiencia del primer amor. Una selección de las composiciones fue publicada en la revista Nueva Educación el año de 1947; dicha selección se encuentra incluida en Nosotros los maestros (2011) y en el tomo 1 de su Obra antropológica (2012). 
escolares evidencia que Arguedas "no solo está enseńando e investigando, está trabajando por un cambio radical en el modelo de la educación peruana, sobre todo en la sierra sur" $(1999,15)$.

\section{LAS POESÍAS LírICAS: LA EXPRESIÓN DE LAS VIVENCIAS DE LOS ALUMNOS}

Arguedas estimula la composición literaria entre sus alumnos a partir de fechas significativas del calendario escolar con el fin de conocer y apreciar la expresión de sus vivencias y de su alma creativa. El Día del Indio es el tema propuesto como motivo de inspiración de los estudiantes, quienes escriben poesías y breves textos narrativos sobre dicho asunto. Las composiciones escritas confirman la capacidad creativa de los alumnos indios y mestizos del Colegio Pumaccahua, a la vez que demuestran la calidad poética de la lengua quechua cuya fuerza expresiva e influencia se percibe en el castellano de los alumnos ${ }^{10}$.

Los textos líricos que figuran en la revista Pumaccahua corresponden a los estudiantes Blas Aguilar, Arturo Castro, Mario Aragón y Alberto Caballero. Las poesías merecen la valoración de Arguedas, quien no duda en reconocer las condiciones literarias de sus autores, en especial del alumno

10 En el artículo "Entre el kechwa y el castellano. La angustia del mestizo", que data de septiembre de 1939, Arguedas afirma que la influencia del quechua es un rasgo dominante en el habla de los indios y mestizos, tal como lo observa entre sus alumnos: "Estamos asistiendo aquí a la agonía del castellano como espíritu y como idioma puro e intocado. Lo observo y lo siento todos los días en mi clase de castellano del colegio Mateo Pumaccahua, de Canchis. Mis alumnos mestizos, en cuya alma lo indio es dominio, fuerzan el castellano, y en la morfología íntima de ese castellano que hablan y escriben, en su sintaxis destrozada, reconozco el genio del kechwa” (2012, T.1, 208). 
Blas Aguilar, de quien la publicación incluye cuatro bellas poesías. El autor de Diamantes y pedernales aprecia su talento creativo y avizora un promisorio futuro literario dadas sus singulares condiciones equiparables a las de los mejores poetas peruanos:

Es posible que este niño llegue a ser uno de los poetas más notables de su generación. Sabe tomar las palabras en su expresión absoluta; y les da, a veces, el infinito sentido de la belleza que tienen las palabras. Esto parece que lo hubiera oído de Eguren o de Emilio Adolfo Westphalen (Arguedas, $2018,5)^{11}$.

La calidad poética de los versos del adolescente Blas Aguilar se revela en los poemas "Aldea indígena en fiesta", "Pomacanchi", "Quena" y "Mi pueblo". Los textos líricos se refieren a pasajes de la vida del pueblo desde la perspectiva del alma de un adolescente y demuestran un dominio del lenguaje poético y de los recursos estilísticos propios de la poesía. El poema "Aldea indígena en fiesta" trata sobre la algazara que despierta la llegada de la fiesta en un pueblo local. La voz poética hace oscilar el sentimiento del indio entre la pena y la alegría, aunque la emoción de la fiesta se cierne paulatinamente sobre el pueblo. Mediante bellas imágenes, el yo lírico anuncia la llegada de las primeras luces del día:

11 Luego de terminado el periodo escolar, varios de los alumnos de Arguedas destacaron en el campo de la actividad literaria. Arturo Castro y Blas Aguilar cultivaron la creación poética y desarrollaron una notable producción. Castro obtuvo importantes premios en concursos literarios en Cusco y fue considerado un poeta de gran calidad; Aguilar continuó escribiendo poesías hasta que la muerte acabó con su actividad creadora en un momento de madurez. Demetrio Zamalloa, otro alumno de Arguedas en el Colegio Pumaccahua, también componía versos de esmerada calidad poética (Osorio, 2018). 
Amanecer torrente de oro y plata

filón de alegría, seguido por el grito del indio (Arguedas, 2018, 5).

Luego, en ese escenario, el sonido de las campanas denota el comienzo de la fiesta:

Las campanas del villorrio tañen y sus extinguidas notas ya llegan $\mathrm{y}$ anuncian que es fiesta (Arguedas, 2018, 5).

Creado el ambiente propicio para la emoción colectiva, la tristeza da paso a la alegría, que se apodera del alma del indio:

Al compás de la música del viento, el indio extingue su tristeza, su alma prorrumpe en gritos de alegría, y su risa ya es ahora, la melancólica luz de la luna, que se filtra en el vidrio de su dolor (Arguedas, 2018, 6).

Apelando al símil y a la metáfora, los versos de Blas Aguilar manifiestan que la alegría y el gozo son también parte de la vida del poblador del ande, tal como lo hace saber al niño indio que va a la fiesta impulsado por el sonido de las quenas, los tambores y los bombos que despiertan su mente:

Niño indio de las punas,

bajas con paso lento a la fiesta que hay en tu aldea, tus ojitos, brillan de alegría como dos perlas tus pupilas amarillentas y negras indican que en tu alma hay todavía vida (Arguedas, 2018, 6).

El talento poético de Blas Aguilar se reafirma en los conmovedores versos de la elegía Pomacanchi, que escribe después de que dicho caserío fuese completamente destruido por un terremoto el 24 de junio de 1939. El 
poema está articulado sobre la oposición vida-muerte, que contrapone dos momentos en la historia del pueblo de Pomacanchi: el periodo de solaz y felicidad anterior al terremoto, y el estado de desolación y destrucción en que se encuentra después de ocurrido el fenómeno sísmico.

En versos de hondo sentir, la voz lírica del poema testimonia el instante en que se produce el violento sismo, así como su estrépito y fuerza destructiva:

Pueblo dormido bajo la sombra del olvido.

Fue despertado por el grito mismo de sus gentes.

El ruido del terremoto en el Ande majestuoso resonó

y el sacudir del hijo de la Desolación

rompió el cuerpo del pueblo, hasta entonces sólo mecido

por la brisa de su azulada laguna (Arguedas, 2018, 6).

El poema testimonia el enorme impacto del terremoto que ha dejado al pueblo sin ningún signo de vida. El gemido y el llanto expresan un hondo pesar:

El paisaje de tu vida gime, llora y se lamenta

perdido te encuentras bajo el escombro de tu suspiro (Arguedas, 2018, 6).

No solo el paisaje de Pomacanchi, sino también la campana, que otrora llamaba al pueblo, se halla en doloroso silencio:

La campana de la iglesia llora en silencio la muerte de la melancólica torre y ya no tańe, solo llora (Arguedas, 2018, 6).

El templo en el que se congregaban los fieles del lugar se halla, igualmente, destruido: 
La iglesia ha muerto en las sombras de las noches de otoño su yerto cuerpo se halla adolorido por el viaje que ha emprendido a las regiones del olvido (Arguedas, 2018, 7)

En el pueblo, solo se escucha llanto por doquier y la desolación dominante en la escena revela la desgracia que castiga duramente al caserío de Pomacanchi. Su fisonomía se ha trastocado por completo y, para denotar dicha calamidad, Blas Aguilar apela al contraste, que le permite mostrar la radical transformación experimentada por el pueblo. La plaza del pueblo “juntamente con las flores, llora”; antes "era un rincón de recias risas”. Asimismo, el parque que era "copia de la alegría de un pueblo / es tierra donde sólo hay muertos". Las calles de la localidad "lloran, golpeadas por la pared de las casitas" $(2018,7)$. El llanto, el quejido y el dolor se reiteran en los versos de la elegía, lo que intensifica aún más el sufrimiento de un pueblo que no encuentra consuelo para su desgracia.

En ese mundo derruido, donde la muerte se ha impuesto sobre la vida, el divino astro, símbolo característico del imaginario andino, puede llegar a convertirse en una entidad de significado negativo:

No siempre la suave risa del Sol es alegre también es rayo de tristeza y cruz de olvido (Arguedas, 2018, 7).

Al ocultarse, el Sol dice que "la historia de un pueblo ha concluido con un canto de tristeza" $(2018,7)$. Además de esta conmovedora elegía, la calidad poética de Blas Aguilar se puede apreciar en los versos de otros dos poemas de su autoría: "Quena”, que describe el sentimiento que produce en su alma la melodía del instrumento del arte musical andino, y "Mi pueblo", en el 
que hace una invocación para que retorne la vida y la alegría a su pueblo natal en lugar de la tristeza, la pena y el olvido que reinan en él.

\section{La prosa narrativa: LA CULTURA local como temática de InSPRACIÓN}

Los textos narrativos publicados en la revista Pumaccahua son breves, guardan estrecha relación con los temas tratados en las poesías, dan a conocer las reflexiones de los alumnos sobre determinados aspectos de la vida de la población indígena y contienen opiniones referidas a la situación del indio. Sobre estas composiciones, Arguedas precisa: "Casi todos hacen relato y cuentan cómo pasa la vida el pueblo indio, y después opinan” $(2019,10)$.

Reconociendo los méritos literarios de dichas composiciones, el estudioso del mundo andino ve en ellas:

(...) una promesa de buena literatura para el país, y encontramos la expresión de una generación de mestizos más uniforme y más definida, de una juventud que probablemente tendrá una mentalidad y un concepto de nuestra realidad, mucho más uniforme y más segura (Arguedas, 2018, 10).

Los textos narrativos incluidos en la revista pertenecen a los alumnos Lucio Ricasca, Crisólogo Aparicio, Justo Ruelas, Adrián Tairo y Adrián Quispe Alanoca. La narración del estudiante Lucio Ricasca focaliza su interés en la tristeza del indio que encuentra en la música de la quena su permanente compañía, así como en las condiciones de pobreza material en que se halla el indio a pesar de haber pertenecido antiguamente a un imperio poderoso. El texto del estudiante Lucio Ricasca reconoce el valor 
de la cultura aborigen y su aporte en la formación del espíritu mestizo, ya que "en nuestras venas corre la sangre india”; por esa razón, el alumno, en virtud de una sincera identificación con la propia raza nativa, exhorta a exteriorizar nuestra adhesión al pueblo aborigen, porque "el indio es nuestra sangre" $(2018,12)$.

Por otro lado, el texto de Crisólogo Aparicio aborda la faena del indio, los roles que cumplen el hombre y la mujer en el seno de la familia campesina y el término de la jornada con la llegada del atardecer. Vinculada con este texto, se encuentra la narración del estudiante Justo Ruelas, que relata los preparativos que realiza un hombre del campo que va a emprender un viaje hacia otros caseríos: "El indio echa carga a las llamas, empieza el viaje, arreando sus llamas; poco a poco se va alejando, tocando su triste quena” $(2018,12)$.

Otro tema que permite formarse una idea de la vida del pueblo indomestizo se desarrolla en el texto del alumno Adrián Tairo, quien nos transmite sus impresiones sobre la llegada del anochecer. Al ocultarse el Sol, el estudiante describe la escena:

(...) el ambiente se vuelve pálido y sopla un viento desanimado; la noche marca un cambio en el movimiento de la vida local, pues se cierran las tiendas y la plaza se torna vacía porque los pobladores se dirigen presurosos a sus chozas para poder guarecerse (Arguedas, 2018, 12).

La narración del alumno Adrián Quispe Alanoca ilustra la dificultad del pueblo indio de expresarse en el idioma castellano que Arguedas observó en el aula de clases. En una sintaxis fragmentada, que revela la influencia de la 
lengua materna, el estudiante narra la historia de la muerte de un indio y la pena que acongoja al negro Chhuko, amigo del fallecido, quien llora por su muerte ${ }^{12}$. El hijo del indio muerto lo busca, pero sus esfuerzos resultan en vano. Un sentido mítico - que es connatural al pensamiento del mundo aborigen- aflora en el texto, ya que la voz narrativa se identifica con un ave que vuela escuchando "el silbo que produce el viento, el precipicio de la lluvia entre truenos y relámpagos, pues soy el pájaro triste de la puna que tanto lloro entre cerros de nieve perpetua" $(2018,13)$.

\section{LOS TEXTOS DE RECOPILACIÓN SOBRE EL FOLCLOR LOCAL Y REGIONAL}

La revista Pumaccahua ofrece una muestra representativa de expresiones del folclor de la región del sur del Perú obtenida gracias a la recopilación de expresiones musicales, literatura oral, ritos y festividades locales realizadas por los alumnos. Dicho trabajo representa la validación de la etnografía como disciplina científica que permite acercarse al saber tradicional de los pueblos para conocerlo y describirlo.

Arguedas incluye en la revista Pumaccahua los huainos "Charango" y "La leyenda de la Kqantuta” de Alfredo Macedo, escritor del pueblo de Ayaviri,

12 En junio de 1941, Arguedas publica en La Verdad de Sicuani los poemas ganadores del concurso que organizó entre sus alumnos con motivo del Día del Indio. Son de mucho interés sus apreciaciones sobre el proceso de aprendizaje de Quispe Alanoca en la expresión del castellano escrito: "Quispe Alanoca es un poeta de nacimiento; después de un angustioso período de aprendizaje del castellano, durante el cual, con un admirable esfuerzo, con un esfuerzo desesperado, pudo lograr el dominio de nuestro idioma nacional, del gran idioma de Cervantes y Manrique, empezó a crear poemas de una pura y extraordinaria belleza” (Pinilla, 2000, 59). 
y "Pomacanchi" de Gabriel Aragón, músico popular de Canchis"13. También figuran el cuento "El Jhakakllo", la explicación de costumbres tradicionales como "La danza de los Ccanchis", "El oveja velacuy" y "El challakuy"; la descripción de la celebración del Día de Difuntos en los pueblos de San Pablo y Langui, y de la realización de la cosecha de trigo en Canchis.

Los huainos publicados en la revista Pumaccahua pertenecen a la región del sur y, para Arguedas, "son de un valor poético muy superior al de las mulizas del Centro" $(2018,9)$. Apreciando la autenticidad de las canciones del mundo andino, el escritor los considera como "expresión del pueblo y voz del pueblo", pues son "los verdaderos". Desde el punto de vista del escritor, los huainos de dicha región conservan la virtud de la música popular y el alma india, que los inspiran y son su fuente de origen.

En las letras del huaino "Charango" de Alfredo Macedo, el cantor destaca la compañía del instrumento musical, "de mis amarguras / confidente", ante la persecución que sufre el indio "sin que la justicia / nos haga justicia" $(2018$, 9). El contenido del huaino "La leyenda de Kqantuta", también de su autoría, se remonta a la época incaica y tiene como protagonista a una

13 Alfredo Macedo y Gabriel Aragón eran músicos y también compositores de piezas teatrales. En el artículo "La canción popular mestiza e india en el Perú, su valor documental y poético", publicado en agosto de 1940 en el diario La Prensa de Buenos Aires, Arguedas menciona el huaino anónimo y el huaino que tiene la firma de sus autores, como Kilko Waraka, Gabriel Aragón, Francisco Gómez de Negrón, Edmundo Delgado y Alfredo Macedo. En ellos, "el mestizo empieza a ser poeta visible y famoso en su provincia”; en sus huainos "el alma del mestizo, guía del pueblo andino del Perú, está tan clara y tan visible como el alma popular de todos los tiempos en el wayno anónimo" (2012, T.1, 277). En particular, Arguedas valora las condiciones creativas, poéticas y musicales de Gabriel Aragón en el artículo "Gabriel Aragón, poeta popular e intérprete del pueblo andino", publicado en La Verdad de Sicuani en noviembre de 1939. 
bella princesa a quien "el hijo de un jefe / real del Imperio / reclama tu amor"; ello desencadena una búsqueda al término de la cual "eres prisionera / princesa puñera" $(2018,9)$. El tema del huaino "Pomacanchi" de Gabriel Aragón describe poéticamente la destrucción del caserío debido al terremoto de 1939. En letras que denotan sentida pena, el huaino describe la pérdida de alegría, sueños y amores en Pomacanchi. Los versos finales de la canción aluden a la desgracia a través de la imagen de la soledad de las aves del lugar: "Pobres avecillas / ya no tienen vuelo / porque han perdido / todo su consuelo" $(2018,9)$.

Entre las manifestaciones de la literatura oral recogidas por los alumnos, figura el cuento "El Jhakakllo", recopilado por Roberto Mendoza ${ }^{14}$. Este relato ilustra el esquema de los motivos que suelen organizar la estructura temática de la narración folclórica tradicional. En el cuento, se desarrollan dos conocidos motivos: la falta que comete el protagonista de la narración al sucumbir ante la soberbia y el castigo que merece tal acto transgresor. El cuento trata sobre el encargo que le da el Dios Inti al ave llamada "jhakakllo" para que divulgue entre los hombres de la tierra que solo deben comer dos veces al día, pero, en un acto de soberbia por sentirse elegida por la suprema divinidad, el ave cae en la desobediencia y proclama que los hombres deben alimentarse tres veces al día. En respuesta, el Dios Inti lo castiga afeando su

14 El relato es una variante del cuento "El chihuaco mentiroso", que figura en Cuentos peruanos de Arturo Jiménez Borja (1937). En la literatura andina, las aves son protagonistas de conocidas fábulas y narraciones folclóricas. Sergio Quijada estudia este tema en el artículo "Las aves en el folklore de la sierra central" (1969-1970) y la presencia del chihuaco en la tradición oral en su trabajo El chihuaco en el folklore (1954). 
hermoso plumaje; como consecuencia de ello, la desobediente ave "[h]oy llora maldiciendo la hora en que había mentido, y llora con voz fea lamentando en todo el mundo su desdicha” $(2018,14)$.

En la línea del rescate de las tradiciones populares, los alumnos de Arguedas realizan, asimismo, acertadas descripciones de tres expresiones musicales del folclor de la región del Cusco: "La danza de los Ccanchis", "El oveja velacuy" y "El challakuy". La presentación de la primera, realizada por el estudiante Juan Delgado, es una información didáctica e ilustrativa sobre una de las expresiones tradicionales del pueblo de Canchis. Baile que, según sus cultores, se remonta a la época incaica, tenía solo protagonistas indios, los "Camayocc", en un primer momento hasta que se incorporaron personajes de origen espańol, como el Misti, el Doctor o el Soltacho, que, en el desarrollo de la danza, se encuentran sometidos al Alcalde, personaje de condición india. En la descripción de la danza, llama la atención la ridiculización con que son presentados los personajes blancos y el sometimiento a la autoridad del Alcalde local durante el baile.

Esta condición revela la respuesta creativa de los pueblos de la sierra del Perú frente a la imposición hispánica, lo que explica la inversión de la dominación en la ficción representada en la danza. De este modo, la danza, apelando a la ironía y a la burla, nos informa sobre la actitud del pueblo frente a la exclusión y explotación sufridas por los indios con la llegada de los españoles ${ }^{15}$. Esa misma apreciación ayuda a comprender por qué el Soltacho,

15 La danza que se describe ilustra el sentido del humor andino y la función de la parodia en la caracterización de personajes de origen español que se asocian con el poder económico y el abuso 
el cholo que ha ido al servicio militar, es también presentado en términos de risa y gracia. Al respecto, al estar asociado el soldado en el imaginario andino con la represión y el poder, es objeto de ridiculización.

\section{LOS COMENTARIOS DE TEXTOS LITERARIOS: EL JUICIO ESTÉTICO DE LOS ESTUDIANTES}

Con el fin de motivar la valoración de la creación poética, Arguedas selecciona un grupo de poesías de José María Eguren y de Luis Valle Goicochea que despertaron un interés por la lectura literaria y el comentario de textos entre sus alumnos ${ }^{16}$. De modo particular, la poesía de Eguren, poblada de personajes de la imaginación, de seres alados, de protagonistas de mundos de ensueño, atrae la atención de los escolares del Colegio Pumaccahua, "quienes no se cansaban de preguntar detalles de su vida". Luego de la lectura de los textos poéticos de ambos autores, el autor de Yawar fiesta les propone

del sistema de justicia en los pueblos de la sierra del Perú. En el artículo "La fiesta de la cruz. La danza de los sijllas", publicado en julio de 1941 en La Prensa de Buenos Aires, Arguedas explica que -al igual que la danza de Canchis- la danza de los sijllas, también del Cusco, es una muestra de la respuesta "más aguda" y "vengativa" que realiza la comunidad india de la justicia española y republicana.

16 La lectura de textos de autores de la poesía contemporánea en la época en que Arguedas desarrolla su trabajo con los escolares en Sicuani demuestra su interés en motivar el acercamiento a diversas tendencias literarias, así como a la obra de autores peruanos y a la literatura de otros países. En su correspondencia con Westphalen, le comenta sobre el gusto de los alumnos por la poesía peruana y, en particular, de la propia obra poética de este: "Mientras yo aquí leo Eguren, leo Abolición, leo García Lorca con mis alumnos. Y ellos entienden y repiten los poemas cuatro y cinco veces. $\mathrm{Si}$ vieras cuantos ratos de hermosura he pasado con ellos leyendo tus versos y los de Eguren. Y no sólo eso en clase; hay como siete u ocho que vienen a mi casa, y se van a la chacra con tus libros, con el de Enmanuel o Eguren. Después regresan, como a la hora o más, y conversamos en mi cuarto hasta bien entrada la noche" (Westphalen Ortiz, 2011, 54). 
escribir sobre sus impresiones: “(...) todos se alegraron, arrancaron las hojas más limpias de sus cuadernos para decir lo que pensaban sobre los poemas de Eguren" $(2018,25)^{17}$.

Los comentarios que realizan los alumnos sobre la poesía de Eguren ilustran una variedad de respuestas emotivas que produce en ellos la calidad lírica del autor de Simbólicas. El conocido poema "El bote viejo", por ejemplo, suscita una honda admiración en los alumnos Héctor Núñez, Teodoro Ramos y Oscar Mesa. Una experiencia similar se encuentra en los estudiantes Edgardo Ochoa, quien nos dice que "casi el total de sus versos han quedado grabado en mi pensamiento", y Cecilio Kenkuri, quien al relatar el contenido del poema lo recrea y escribe una nueva versión en prosa. Entre los escolares, Modesto Zapata, quien prefiere los poemas "El dolor de la noche" y "El cuarto cerrado", confiesa su identificación con los sentimientos que expresa el yo lírico en las poesías de Eguren como si él fuese el poeta y el autor, su alter ego: "Estas poesías parece que no salen del corazón y del sentimiento del poeta sino que salen de mí y yo soy el que estoy haciendo de cuenta que describo y siento de las cosas vistas o imaginadas por él" $(2018,25)$.

17 Además de la lectura y comentario de los poemas de Eguren y Valle Goicochea, la poesía de César Vallejo también fue un tema de interés de los alumnos en las clases de Arguedas. En una de sus misivas a Moreno Jimeno, el escritor le comenta la sentida emoción con que compartió la lectura de sus versos con los estudiantes: “(...) he dado una clase especial, en todas las secciones del colegio sobre Vallejo. Les hablé de la vida de Vallejo, bastante largo, de todos sus trabajos, de sus luchas, y de esto en detalle. Y terminé leyendo algunos de sus poemas publicados en Garcilaso". Basándose en la lectura de un artículo del propio Moreno Jimeno sobre Vallejo comentada en clases, afirma: "Los muchachos saben ahora, perfectamente quién fue Vallejo, y estoy seguro que lo quieren" (Forgues, 1993, 21). 
La capacidad lúdica de las composiciones se revela también en la breve carta que le escribe el alumno Jorge Castillo a Eguren, en la que enfatiza la bondad y ternura del poeta, y la conmoción que le produce la lectura del poema "El cuarto cerrado". Otros poemas de Eguren, como "El dolor de la noche", "La nave enferma" y "Lied III", son, igualmente, muy valorados por los adolescentes, a la vez que les produce un gusto estético y los emocionan.

Otro género que es objeto del acertado comentario de los escolares es el narrativo. Para tal fin, Arguedas les plantea la lectura de dos libros: Cuentos peruanos de Arturo Jiménez Borja, publicado en 1937 y cuyo contenido se centra en la temática andina; y Doce novelas de la selva de Fernando Romero, publicado en 1934 y que, conformado por narraciones referidas al mundo amazónico, será conocido más adelante con el título de Doce relatos de la selva. Sobre el primer libro, los juicios de los alumnos toman como referencia la lectura de los cuentos "La achiqué", "El gallinazo y el zorro", "La huachua y el zorro" y "El tigre, la serpiente y la tortuga". Los comentarios sobre el segundo libro se basan en la lectura de "El abrazo", "Una madre", "El leproso" y "La creciente".

En relación con el libro de Jiménez Borja, los comentarios de los alumnos se enfocan en el plano de la expresión, los temas tratados, los personajes, los valores asociados con ellos y el sentido aleccionador de los cuentos, entre otros puntos. Así, el estudiante Paulino Aguilar subraya la sencillez del lenguaje y la naturalidad de la expresión como características positivas de los cuentos recopilados; para Feliciano Bustamante, los cuentos ilustran la capacidad de creación del indio peruano. En su comentario, José Galiano 
destaca la habilidad creativa de los indios, a quienes considera "verdaderos artistas", así como el sentido moral que transmiten los cuentos, elemento importante en la formación de los educandos.

Blas Aguilar observa en los cuentos otros elementos que deben valorarse. Por un lado, con el lenguaje diáfano de su prosa "se expresa claramente el alma serrana"; además, el alumno enfatiza el sentido moral de los cuentos y la simbología de las acciones de los personajes: "En unas ocasiones un animal es más astuto que otro, en otras ocasiones, frente al animal que antes lo tomaba como un tonto, se cree más humilde, pero siempre con traiciones se pone también al nivel que otro" $(2018,28)$. Así, basado en el contenido de los cuentos, deduce que "el bien va adelante, porque las personas que hacen mal a su prójimo salen siempre con alguna desgracia que los lleva a la tumba" $(2018,28)$.

\section{LOS TEXTOS ENSAYÍSTICOS: EL SENTIDO CRÍTICO Y REFLEXIVO}

Los textos ensayísticos que escriben los alumnos de Arguedas tienen una enorme importancia para su formación, porque incentivan su capacidad para opinar con sentido crítico. La revista Pumaccahua comprende una sección que incluye las respuestas de los estudiantes a cuestionarios de exámenes del curso de Geografía humana aplicados por Arguedas durante el desarrollo de sus clases. Mediante extractos de las evaluaciones, que se refieren "a un problema fundamental de algunos pueblos de América”, el escritor ofrece una selección de las respuestas de los alumnos, que "están plenas de una 
profunda convicción y de una absoluta conciencia del problema que tratan" (2019, 30).

Como afirma el autor, los estudiantes "han explicado y fundamentado su propia convicción; porque han escrito sobre un problema que ellos viven, que se refiere a su propia realidad espiritual" $(2018,30)$. Para el conocido novelista, es digno de mérito que los estudiantes indios y mestizos hayan alcanzado una sólida capacidad de exposición reflexiva, porque ello revela no solo su pensamiento crítico, sino que también afirma su identidad. Al respecto, valora con mucho criterio que "sus alumnos han aprendido a analizar libremente los problemas que ellos están en condiciones de analizar, y que están convencidos de las posibilidades de la raza y de la nación a la que pertenecen" $(2018,30)$.

Además de la propiedad en abordar los temas y del rigor en la formulación de las ideas, Arguedas destaca otros aspectos positivos que se observan en las respuestas de los alumnos. Así, de modo particular, al referirse al examen del curso de Geografía humana del estudiante Óscar Vera, comenta que el tema planteado "lo supo explicar con toda claridad y concisión". Es decir, es importante, por un lado, el lenguaje con que se expresa el pensamiento del alumno y, por otro, su capacidad de síntesis en las respuestas.

Podemos formarnos una idea del logro que alcanzan los alumnos de Arguedas en el cultivo del género expositivo mediante las explicaciones que Carlos Mendoza Contreras, Manuel Cueva, Juan Delgado y el ya mencionado Óscar Vera realizan sobre asuntos relacionados con la influencia de las culturas nativas en los países de América, el problema de latifundio en 
el Perú y los inicios del comercio nacional. Las tres cuestiones, en principio, exigen un conocimiento sobre dichos tópicos de parte de los estudiantes, así como una reflexión y un determinado punto de vista. En la selección de las respuestas, se percibe una coincidencia en señalar que los países con mayor influencia nativa son Perú, Bolivia y Ecuador, para lo cual los estudiantes argumentan apelando a razones históricas y culturales: el fuerte arraigo de la cultura prehispánica, la ubicación geográfica de los países que restringe la influencia extranjera y el lugar donde se encuentran los pueblos de cada país, lo que les permite mantener intactas sus costumbres y tradiciones.

Para los alumnos, en contraparte a los países andinos mencionados, la nación argentina disiente de dicha influencia, ya que es un país conformado por inmigrantes y tiene apertura a la cultura europea. También es pertinente señalar que, desde el punto de vista de los alumnos, existe una valoración de la matriz andina como sustento de la cultura americana y del mestizaje cultural como proyección futura de los pueblos de América. Con relación a ello, es muy significativo que los estudiantes consideren a México como ejemplo de un país cuya identidad reside en la gran riqueza y vitalidad de sus culturas nativas.

\section{Conclusiones}

La experiencia pedagógica de Arguedas en el Colegio Nacional Mateo Pumaccahua, de Sicuani, en el Cusco, tuvo un resultado altamente positivo, de lo cual es un testimonio la revista Pumaccahua, que publicó en 1940 y 
que reúne los trabajos de sus alumnos. En la revista, se plasma una nueva concepción de la educación que se enmarca dentro de los objetivos de la escuela activa y de la investigación-acción, que promueven el protagonismo de los estudiantes y se centran en sus intereses. Frente a una educación memorística, descontextualizada y sin mayor relación con la realidad sociocultural de los estudiantes, Arguedas plantea una educación que valore las vivencias de los alumnos y que fomente su expresión personal, así como un mayor conocimiento de la vida social y cultural de la comunidad.

La revista Pumaccahua ofrece una variedad de géneros discursivos, como la poesía lírica, la narración, el comentario de textos, el ensayo y la descripción de tradiciones culturales de la comunidad local. Así, podemos conocer la expresión de la creación personal de los estudiantes mediante la composición de poesías líricas, cuya temática está inspirada en las vivencias de la comunidad y del mundo andino. Igualmente, apreciamos el talento narrativo de los alumnos en textos que recrean una temática similar, así como la sensibilidad estética y el juicio literario mediante la lectura y el comentario de poesías y narraciones de la literatura peruana. A ello se suma la importancia del trabajo etnográfico a través de la investigación de campo y de la descripción de tradiciones y costumbres de los pueblos de la sierra sur del Perú. En tal sentido, el trabajo de Arguedas en Sicuani es un referente que nos permite valorar las posibilidades creativas de los alumnos, su potencial literario, su capacidad artística y reflexiva, y su identificación con el mundo y la cultura andina. 


\section{REFERENCIAS BIBLIOGRÁFICAS}

Arguedas, José María. Pumaccahua. Estudio introductorio de Javier Liendo García.

$2^{\mathrm{a}}$ ed. Edición facsimilar. Lima: Casa de la Literatura Peruana, 2018.

Arguedas, José María. Obra antropológica. 7 t. Lima: Horizonte, 2012.

Arguedas, José María. Nosotros los maestros. Selección, estudio preliminar y notas de Wilfredo Kapsoli Escudero. 2a ed. Lima: Derrama Magisterial, 2011.

Barrantes, Emilio. Folklore de Huancayo. Huancayo: Colegio Nacional de Santa Isabel, 1940.

Cáceres Villa, Guillermo. "Primeros maestros del Colegio Pumacahua". In: Canchis, Revista cultural del Club Provincial Canchis-Lima, 7, 2012, 60.

Cuenca, Ricardo y Pajuelo, Ramón (Eds.). Arguedas. El Perú y las ciencias sociales: nuevas lecturas. Lima: Instituto de Estudios Peruanos y Derrama Magisterial, 2014.

Forgues, Roland. José María Arguedas. La letra inmortal. Correspondencia con Manuel Moreno Jimeno. Lima: Ediciones de los Ríos Profundos, 1993.

González, Hugo. "El modelo de investigación-acción en la práctica docente de Arguedas". In: Cuenca, Ricardo y Pajuelo, Ramón (Eds.). Arguedas. El Perú y las ciencias sociales: nuevas lecturas. Lima: Instituto de Estudios Peruanos y Derrama Magisterial, 2014, 173-193.

González, Hugo. "La experiencia pedagógica de José María Arguedas". In: Pueblo Continente, 22, 2011, 89-92.

Giraudo, Laura. "Un campo indigenista transnacional y 'casi profesional': la apertura en Pátzcuaro (1940) de un espacio por y para los indigenistas". In: Giraudo, Laura y Martín-Sánchez, Juan (Eds.). La ambivalente historia del indigenismo. Campo interamericano y trayectorias nacionales 1940-1970. Lima: Instituto de Estudios Peruanos, 2011, 21-98. 
Giraudo, Laura y Martín-Sánchez, Juan. La ambivalente historia del indigenismo. Campo interamericano y trayectorias nacionales 1940-1970. Lima: Instituto de Estudios Peruanos, 2011.

Jurado Párraga, Raúl. "Etnoliteratura y educación en José María Arguedas". In: Demiurgo, Revista de la Universidad Nacional de Educación La Cantuta, 1, 2003, 75-78.

Kapsoli, Wilfredo. "El maestro José María Arguedas”. In: Arguedas, José María. Nosotros los maestros. 2a ed. Lima: Derrama Magisterial, 2011, 17-58.

Mathews, Daniel. La paideia retrógrada: novela y escuela en Arguedas. Huancayo: Centro Cultural José María Arguedas, 1999.

Muñoz, Fanni. "La impronta de Arguedas en la construcción de la ética del funcionario público”. In: Pinilla, Carmen María (Ed.). Arguedas y el Perú de hoy. Lima: Casa Sur, 2005, 189-200.

Osorio, Juan Alberto. "Sobre José María Arguedas en Sicuani”. Lima, 01 sept. 2018. Disponible en: https://creacionheroica1928.blogspot.com/2018/09/ testimonio.html (Fecha de consulta: 20/09/19).

Pinilla, Carmen María. Dos amautas. Arguedas y Barrantes. Lima: Academia Peruana de la Lengua, 2012.

Pinilla, Carmen María. (Ed.). Arguedas y el Perú de hoy. Lima: Casa Sur, 2005.

Pinilla, Carmen María. "Las huellas de Arguedas en La verdad de Sicuani entre 1941 y 1942". In: Cuadernos Arguedianos, 3, 2000, 57-68.

Vadillo Villa, José. "El rastro de Arguedas". Variedades, suplemento de El Peruano, Lima, 23 de mayo de 2011, 5-7.

Westphalen Ortiz, Inés (Comp.). El rio y el mar. Correspondencia José María Arguedas / Emilio Adolfo Westphalen (1939-1969). México D.F.: Fondo de Cultura Económica, 2011. 